\title{
Evidence-based recommendations for energy intake in pregnant women with obesity
}

\author{
Jasper Most, ${ }^{1}$ Marshall St Amant, ${ }^{2}$ Daniel S. Hsia, ${ }^{1}$ Abby D. Altazan, ${ }^{1}$ Diana M. Thomas, ${ }^{3}$ L. Anne Gilmore, ${ }^{1}$ Porsha M. Vallo, ${ }^{1}$ \\ Robbie A. Beyl, ${ }^{1}$ Eric Ravussin, ${ }^{1}$ and Leanne M. Redman ${ }^{1}$ \\ 'Reproductive Endocrinology and Women's Health, Pennington Biomedical Research Center, Baton Rouge, Louisiana, USA. ²Maternal and Fetal Medicine, Woman's Hospital, Baton Rouge, Louisiana, USA. \\ ${ }^{3}$ United States Military Academy, West Point, New York, USA.
}

\begin{abstract}
BACKGROUND. In women with obesity, excess gestational weight gain ( 2270 g/week) occurs in 2 out of 3 pregnancies and contributes to metabolic impairments in both mother and baby. To improve obstetrical care, objectively assessed information on energy balance is urgently needed. The objective of this study was to characterize determinants of gestational weight gain in women with obesity.
\end{abstract}

\begin{abstract}
METHODS. This was a prospective, observational study of pregnant women with obesity. The primary outcome was energy intake calculated by the energy intake-balance method. Energy expenditure was measured by doubly labeled water and whole-room indirect calorimetry and body composition as a 3-compartment model by air displacement plethysmography and isotope dilution in early (13-16 weeks) and late (35-37 weeks) pregnancy.

RESULTS. In pregnant women with obesity $(n=54)$, recommended weight gain $(n=8,15 \%)$ during the second and third trimesters was achieved when energy intake was $125 \pm 52 \mathrm{kcal} / \mathrm{d}$ less than energy expenditure. In contrast, women with excess weight gain (67\%) consumed $186 \pm 29 \mathrm{kcal} / \mathrm{d}$ more than they expended $(P<0.001)$. Energy balance affected maternal adiposity (recommended: $-2.5 \pm 0.8 \mathrm{~kg}$ fat mass; excess: $+2.2 \pm 0.5$; inadequate: $-4.5 \pm 0.5 ; P<0.001$ ) but not fetal growth. Weight gain was not related to demographics, activity, metabolic biomarkers, or diet quality. We estimated that energy intake requirements for recommended weight gain during the second and third trimesters were not increased as compared with energy requirements early in pregnancy ( $34 \pm 53 \mathrm{kcal} / \mathrm{d}, P=0.83$ ).
\end{abstract}

CONCLUSION. We here provide what we believe are the first evidence-based recommendations for energy intake in pregnant women with obesity. Contrary to current recommendations, energy intake should not exceed energy expenditure.

TRIAL REGISTRATION. ClinicalTrials.gov, NCT01954342.

FUNDING. This study was funded by the National Institutes of Health (R01DK099175) and the Clinical Research Cores at Pennington Biomedical Research Center (U54GM104940 and P30DK072476).

\section{Introduction}

According to the 2009 Institute of Medicine guidelines, women entering pregnancy with obesity should limit total weight gain to 5-9 kg (1). Despite these recommendations, excess gestational weight gain occurs in 2 out of every 3 pregnancies among women with obesity $(2,3)$, increasing the risks of obesity and type 2 diabetes mellitus in both the mother and baby $(4,5)$. Thus, for women with obesity, effective weight gain management in pregnancy is an unmet need in clinical practice.

Lifestyle modification therapy is considered the first line strategy for controlling gestational weight gain. To date, more than

Delated Commentary: p. 4567

Conflict of interest: The authors have declared that no conflict of interest exists. Copyright: (5) 2019, American Society for Clinical Investigation.

Submitted: May 17, 2019; Accepted: July 23, 2019; Published: September 23, 2019 Reference information: J Clin Invest. 2019;129(11):4682-4690.

https://doi.org/10.1172/JCl130341.
6300 patients who are overweight or obese have participated in effectiveness trials evaluating lifestyle interventions, including LIMIT (6), DALI (7, 8), UPBEAT (9), RADIEL (10), and LIFEMoms (11). Only half of these studies report a significant reduction in the incidence of excess gestational weight gain $(7,11)$. Overall, reductions in total weight gain were modest and averaged $0.5 \mathrm{~kg}$ (6-11). Very few studies demonstrated downstream improvements in maternal and infant outcomes at birth $(6,8,11)$. The low efficacy of lifestyle intervention trials is generally attributed to poor patient adherence, yet we and others $(6,8-11)$ hypothesize that most interventions are of insufficient intensity. Consequently, they produce only small perturbations in energy balance (intake minus expenditure) and therefore small changes in weight gain.

The Institute of Medicine recommends that all pregnant women increase energy intake by $340-450 \mathrm{kcal} / \mathrm{d}$ during the second and third trimesters (1). Such recommendations for dietary energy intake $(1,12,13)$ were based on studies in pregnant women without obesity (14-16). In recognition of the different needs for the patient with obesity, the American College of Obstetricians 


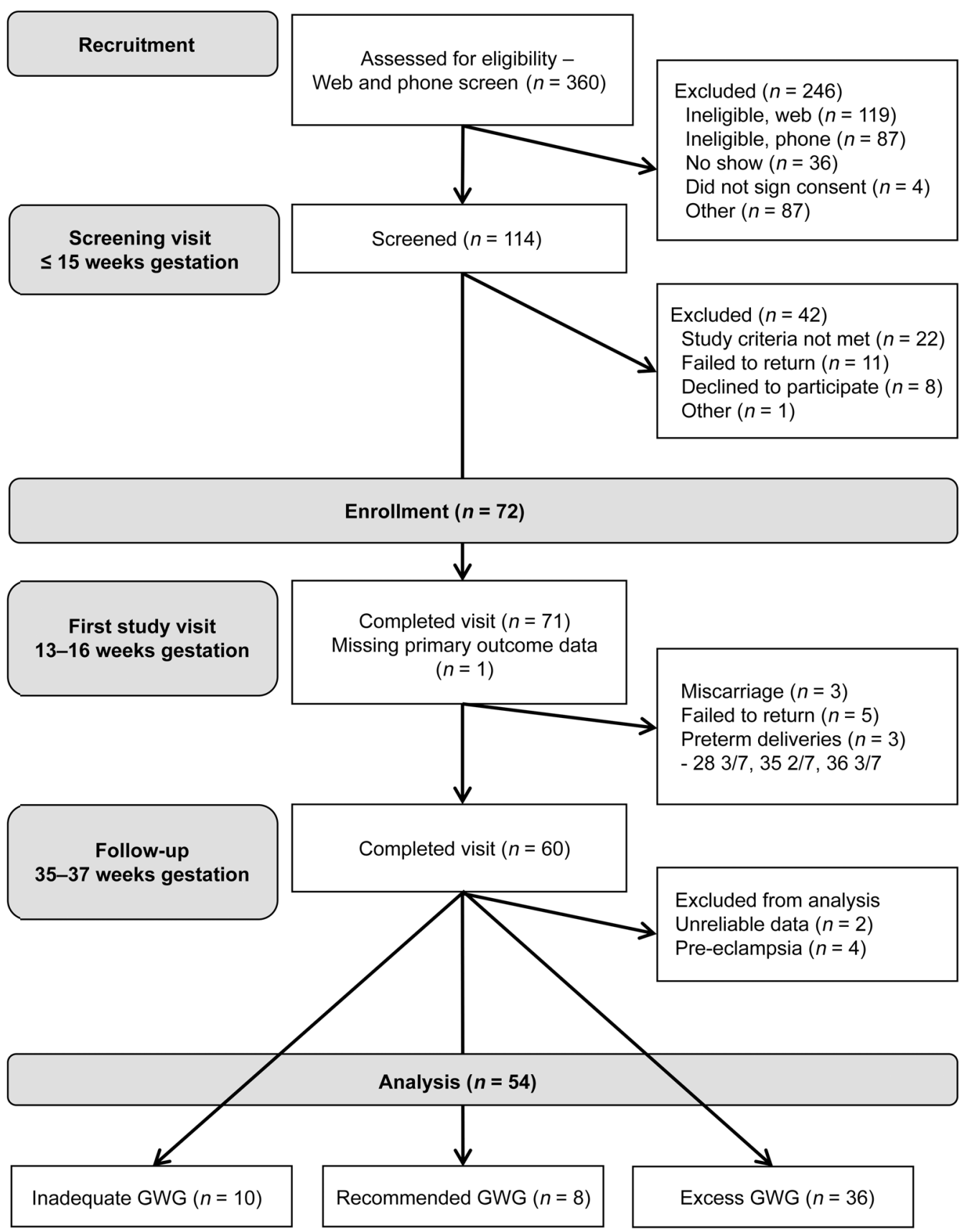

Figure 1. Participant throughput. Seventy-two women were enrolled, 60 completed the late pregnancy assessment, and 54 satisfied the criteria for inclusion in the statistical analysis.

met $(n=22)$

$(n=11)$

articipate $(n=8)$

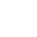




\begin{tabular}{|c|c|c|c|c|}
\hline & INA $(n=10)$ & $\operatorname{REC}(n=8)$ & $\operatorname{EXS}(n=36)$ & $P$ \\
\hline \multicolumn{5}{|l|}{ Maternal demographics } \\
\hline Age, years & $29.2 \pm 1.3$ & $25 \pm 1.7$ & $27.7 \pm 0.8$ & 0.15 \\
\hline Parity, $n(0,1, \geq 2)$ & $3,3,4$ & $4,2,2$ & $16,14,6$ & 0.38 \\
\hline Race, $n$ (African American, White, Others) & $6,3,1$ & $4,3,1$ & $12,22,2$ & 0.41 \\
\hline Education, $n(1,2,3,4)$ & $1,4,2,3$ & $1,3,4,0$ & $6,11,10,9$ & 0.52 \\
\hline Household income, poverty-to-income ratio & $3.5 \pm 0.9$ & $3.0 \pm 0.6$ & $3.8 \pm 0.4$ & 0.66 \\
\hline \multicolumn{5}{|l|}{ Maternal anthropometrics, screening } \\
\hline Cestational age, weeks & $10.2 \pm 0.6$ & $12.2 \pm 0.6$ & $11.5 \pm 0.4$ & 0.15 \\
\hline Body height, m & $1.65 \pm 0.03$ & $1.68 \pm 0.02$ & $1.62 \pm 0.01$ & 0.21 \\
\hline Body weight, kg & $110.1 \pm 7.3^{\mathrm{A}}$ & $97.4 \pm 5.8^{B}$ & $91.9 \pm 1.9^{B}$ & 0.005 \\
\hline Body mass index, $\mathrm{kg} / \mathrm{m}^{2}$ & $40.6 \pm 2.5^{A}$ & $34.6 \pm 1.6^{\mathrm{B}}$ & $34.8 \pm 0.5^{\mathrm{B}}$ & 0.003 \\
\hline Obesity class, $n(1,2,3)$ & $3,2,5$ & $5,2,1$ & $17,17,2$ & 0.009 \\
\hline \multicolumn{5}{|l|}{ Pregnancy outcomes } \\
\hline Gestational diabetes mellitus, $n$ & 1 & 3 & 3 & 0.08 \\
\hline Cestational hypertension, $n$ & 2 & 0 & 2 & 0.21 \\
\hline \multicolumn{5}{|l|}{ Delivery outcomes } \\
\hline Cestational weight gain, kg & $1.5 \pm 0.5$ & $4.5 \pm 0.2$ & $10.3 \pm 0.6$ & $<0.001$ \\
\hline Cestational age, delivery, weeks & $39.5 \pm 0.4$ & $38.8 \pm 0.3$ & $39.6 \pm 0.2$ & 0.14 \\
\hline Anemia, $n$ & 0 & 0 & 2 & 0.60 \\
\hline Labor type, $n(0,1,2)$ & $2,7,1$ & $3,4,1$ & $9,18,9$ & 0.68 \\
\hline Delivery type, $n(1,2,3,4)$ & $5,0,3,2$ & $5,0,0,3$ & $20,0,7,9$ & 0.58 \\
\hline Nonelective cesarean section, $n$ & 5 & 3 & 16 & 0.87 \\
\hline Shoulder dystocia, $n$ & 0 & 0 & 0 & 1.00 \\
\hline $\operatorname{APGAR} 1 \min , n(\leq 7,8,9)$ & $0,5,4$ & $1,4,3$ & $1,16,17$ & 0.40 \\
\hline $\operatorname{APGAR} 5 \min , n(\leq 78,9)$ & $0,0,9$ & $1,0,7$ & $0,2,32$ & 0.17 \\
\hline \multicolumn{5}{|l|}{ Infant outcomes } \\
\hline Birth weight, g & $3475 \pm 104$ & $3541 \pm 190$ & $3439 \pm 81$ & 0.85 \\
\hline Birth length, cm & $50.9 \pm 0.5$ & $51.2 \pm 1.3$ & $50.8 \pm 0.4$ & 0.91 \\
\hline Infant size, $n$ (SGA, AGA, LGA) & $0,9,1$ & $0,6,2$ & $1,28,7$ & 0.87 \\
\hline \multicolumn{5}{|c|}{$\begin{array}{l}\text { According to the } 2009 \text { Institute of Medicine guidelines, weight gain was classified as inadequate (INA, }<170 \\
\mathrm{~g} / \text { week), recommended (REC, } \geq 170 \text { and }<270 \mathrm{~g} / \text { week), and excessive (EXS, } \geq 270 \mathrm{~g} / \text { week). Education is } \\
\text { categorized into high school (1); } 1-3 \text { years of college (2); college degree (3); postgraduate work (4). Labor types } \\
\text { are no labor ( } 0 \text { ); spontaneous/augmented (1); induced ( } 2 \text { ). Delivery types are spontaneous vaginal (1); operative } \\
\text { vaginal (2); caesarean section with labor (3); caesarean section without labor ( } 4 \text { ), LGA, large for gestational age, } \\
\text { SGA, small for gestational age. } P \text { presents the statistical significance of the group comparison, tested using } \\
\text { linear mixed effect models with group used as a covariate (continuous variables) or using } \chi^{2} \text { tests (categorical } \\
\text { variables). } P \leq 0.05 \text { is considered statistically significant. } P \text { values in bold are significant. }{ }^{A, B} \text { Shared letters } \\
\text { indicate no significant differences between groups in post hoc comparison. }\end{array}$} \\
\hline
\end{tabular}

\section{Table 1. Subject characteristics}

mass by $4.5 \pm 0.2 \mathrm{~kg}$ during the observation period. While these women accumulated $7.0 \pm 0.8 \mathrm{~kg}$ of fat-free mass (Figure 2B) that included fetal growth $(2.7 \pm 0.1 \mathrm{~kg})$, the total weight gain was also the result of losing fat mass $(-2.5 \pm 0.8 \mathrm{~kg})$.

In Table 2, results of the primary outcomes - energy intake, energy expenditure and energy deposition - are summarized. Energy intake throughout the second and third trimesters was calculated as the sum of energy expenditure across pregnancy (calculated as mean of total daily energy expenditure early and late in pregnancy), and energy deposition, i.e. changes in fat mass and fat-free mass. Therefore, energy intake is the mean energy intake per day between $14.9 \pm 0.1$ weeks and 35.9 \pm 0.1 weeks of pregnancy. In the REC group, daily energy intake was $2698 \pm 99 \mathrm{kcal} / \mathrm{d}$ and energy expenditure was $2824 \pm 105 \mathrm{kcal} / \mathrm{d}$ (Table 2 and Figure 3A). Therefore, women with recommended weight gain maintained a negative energy balance, i.e. intake minus expenditure, of $-125 \pm 52 \mathrm{kcal} / \mathrm{d}$ during pregnancy (Figure $3 \mathrm{~B}$ ).

Excess weight gain. The EXS group gained $10.3 \pm 0.6 \mathrm{~kg}$ body weight during the observation period $(P<0.0001)$. Compared with the REC group, the difference in total weight gain was not due to the accumulation of fat-free mass, including fetal growth, but to gains in fat mass $(2.2 \pm 0.5 \mathrm{~kg}, P<0.001$, Figure $2 \mathrm{~B})$. For the EXS group, the weight as recommended (REC), 36 women (67\%) gained excess weight (EXS), and 10 (19\%) had inadequate (INA) weight gain (Figure 2A). The groups did not differ by demographic characteristics (Table 1$)$, but the INA group had more severe obesity $(P$ $=0.002$, Table 1) and was more insulin resistant compared with the EXS group $(P=0.008$, Supplemental Table 1 ; supplemental material available online with this article; https://doi.org/10.1172/ JCI130341DS1). In a sensitivity analysis, we excluded the 2 most obese patients (BMI: 57.1, 46.0, both INA group). The differences in obesity and insulin resistance early in pregnancy disappeared, whereas primary outcomes were unchanged. Pregnancy outcomes, delivery outcomes, and infant size at birth were not different among the weight gain groups (Table 1 ).

Recommended weight gain. At a rate of weight gain of $218 \pm 90$ $\mathrm{g} /$ week, women with recommended weight gain increased body energy intake and energy expenditure throughout the second and third trimesters were not significantly different from the REC group ( $P=0.16$ and $P=0.48$, respectively, Table 2 and Figure 2A). However, energy balance was positive $(186 \pm 29 \mathrm{kcal} / \mathrm{d})$ and significantly different from the REC group $(P<0.001$, Figure $2 \mathrm{~B})$.

Inadequate weight gain. In the INA group, women gained only $1.5 \pm 0.5 \mathrm{~kg}(P=0.03)$. While fat-free mass accumulation and fetal growth were comparable with the REC and EXS groups, the INA group lost fat mass $(-4.5 \pm 0.5 \mathrm{~kg}, P=0.10$, Figure $2 \mathrm{~B})$. Energy intake throughout the second and third trimesters was not different from energy intake in the REC group $(P=0.51)$. Energy balance was negative, but the difference to the REC group was not significant $(-262 \pm 32 \mathrm{kcal} / \mathrm{d}, P=0.08$, Figure $2 \mathrm{~B})$.

Physiologic and behavioral determinants of gestational weight gain. The difference in energy balance among the groups was 
A

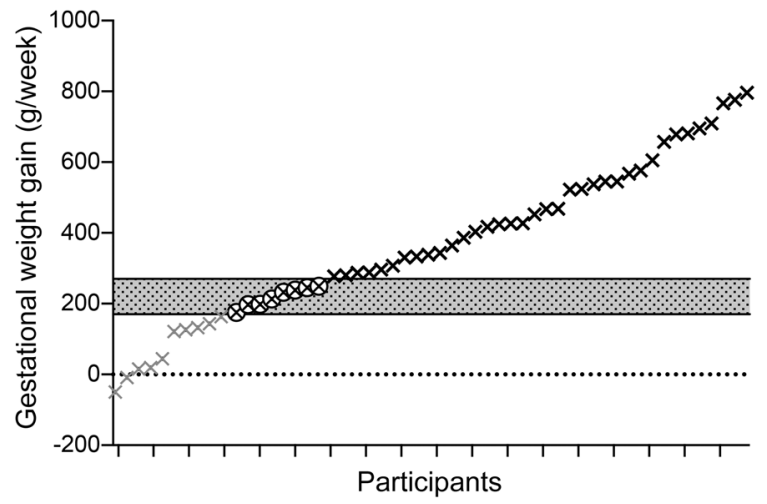

B

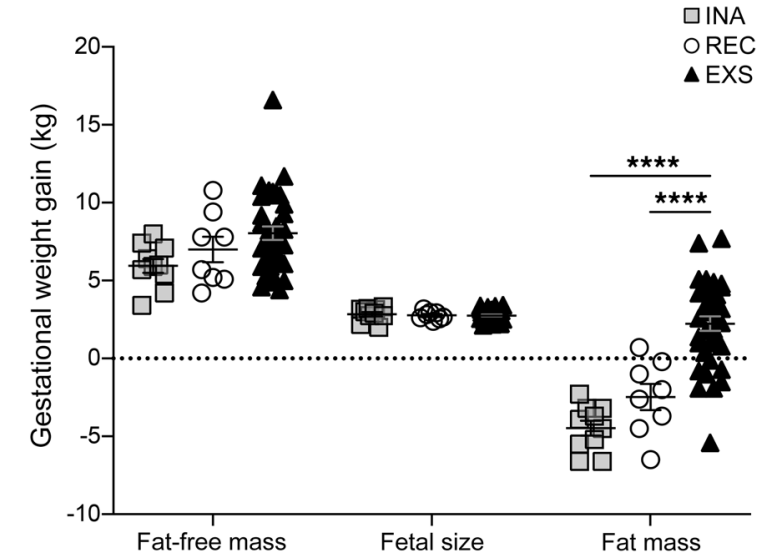

not accounted for by differences in physical activity (Supplemental Table 2). We observed a smaller increase in sleeping energy expenditure in women with excess and inadequate weight gain as compared with women with recommended weight gain (both $P<$ 0.01, Supplemental Table 1). Physiological and behavioral factors including insulin, thyroid hormones, sympathetic nervous system activity and gut hormones, diet quality, and eating behavior constructs, including mindful eating and food cravings, were not different among the weight gain groups (Supplemental Tables 1-4).

Energy intake for recommended gestational weight gain. Using our data, we developed a linear regression equation to estimate the change in energy intake from early pregnancy to the second and third trimesters of pregnancy required to achieve recommended gestational weight gain. This approach assumed that energy intake requirements early in pregnancy were equivalent to total daily energy expenditure $(1,13,14)$ and energy intake requirements during the second and third trimesters equal energy intake in women with recommended weight gain. Thus, the change in energy intake was calculated as difference between measured energy intake during pregnancy and total daily energy expenditure early in pregnancy. Using an observed gestational weight gain (GWG) as an independent variable, the
Figure 2. Rate and composition of weight gain. (A) Gestational weight gain is shown as grams per week for each individual participant. According to 2009 Institute of Medicine guidelines, weight gain was classified as inadequate (in gray, INA, $<170 \mathrm{~g} /$ week, $n=10$ ), recommended (in white, $\mathrm{REC}, \geq 170 \mathrm{~g} /$ week and $<270 \mathrm{~g} /$ week, $n=8$ ), and excessive (in black, EXS, $\geq 270 \mathrm{~g} /$ week, $n=36$ ). The highlighted box shows the weight gain recommendations. (B) Data shown represent change from early (14.9 \pm 0.1 weeks) to late pregnancy ( $35.9 \pm 0.1$ weeks) and are presented for each individual and as mean \pm SEM for fat-free mass, fetal size, and fat mass, as observed in kilograms. Differences among weight gain groups were tested using linear mixed effect models with group used as a covariate. ${ }^{* * *} P<0.001$ of the post-hoc comparison between groups of the linear mixed model.

change in energy intake (Change EI) can be estimated as: Change EI $[\mathrm{kcal} / \mathrm{d}]=1.178 \pm 0.108^{*} \mathrm{GWG}$ [g/week] $-225 \pm 46 ; \mathrm{R}^{2}=0.70$.

The Change EI is relative to energy intake and energy expenditure in early pregnancy and GWG is rate of gestational weight gain during second and third trimesters (Figure 4A). To achieve the recommended rate of weight gain suggested by the Institute of Medicine for women with obesity (170-270 g/week), the change in energy intake during the second and third trimesters would need to be between $-25 \pm 46 \mathrm{kcal} / \mathrm{d}$ to $+93 \pm 46 \mathrm{kcal} / \mathrm{d}$. In Figure $4 \mathrm{~B}$, we estimated changes in energy intake for published weight gain data in lifestyle intervention studies. Our model of estimated energy intake showed that patients in the intervention groups consumed $196 \pm 20 \mathrm{kcal} / \mathrm{d}$ more than early pregnancy energy requirements (range +78 to $+310 \mathrm{kcal} / \mathrm{d}$ ). Control groups in these respective studies consumed higher energy intakes $(334 \pm 32 \mathrm{kcal} / \mathrm{d})$ during the same time period (data not shown).

\section{Discussion}

We believe that this is the first study to provide evidence-based recommendations for energy intake in pregnant women with obesity that are in contrast to current recommendations by the Institute of Medicine. To deliver such recommendations, we describe phenotypic, behavioral, and metabolic characteristics and determinants of gestational weight gain exclusively in women with obesity using a comprehensive battery of objective measures per-

\section{Table 2. Energy intake, energy expenditure and energy deposition in pregnant women with obesity}

$\begin{array}{lcccc} & \text { INA }(\boldsymbol{n}=10) & \text { REC }(\boldsymbol{n}=\mathbf{8}) & \text { EXS }(\boldsymbol{n}=36) & \boldsymbol{P} \\ \text { Energy intake, kcal/d } & 2581 \pm 132^{\mathrm{A}} & 2698 \pm 99^{\mathrm{A}, \mathrm{B}} & 2908 \pm 64^{\mathrm{B}} & \mathbf{0 . 0 4} \\ \text { TDEE, early pregnancy, kcal/d } & 2719 \pm 142 & 2664 \pm 119 & 2563 \pm 53 & 0.41 \\ \text { TDEE, late pregnancy, kcal/d } & 2966 \pm 156 & 2984 \pm 121 & 2882 \pm 54 & 0.92 \\ \text { Mean pregnancy TDEE, kcal/d } & 2842 \pm 137 & 2824 \pm 105 & 2722 \pm 48 & 0.48 \\ \text { Energy deposition, kcal/d } & -262 \pm 32^{\mathrm{A}} & -125 \pm 52^{\mathrm{A}} & 186 \pm 29^{\mathrm{B}} & <\mathbf{0 . 0 0 0 1}\end{array}$

Data are presented as mean \pm SEM. According to 2009 Institute of Medicine guidelines, weight gain was classified as inadequate (INA, $<170 \mathrm{~g} /$ week), recommended (REC, $\geq 170 \mathrm{~g} /$ week and $<270 \mathrm{~g} /$ week), and excessive (EXS, $\geq 270 \mathrm{~g} /$ week). Energy intake is calculated as mean pregnancy TDEE + energy deposition, in which energy expenditure is calculated as mean TDEE in early (14.9 \pm 0.1 weeks) and late pregnancy ( $35.9 \pm 0.1$ weeks), and energy deposition is calculated based on the changes in fat mass $(9500 \mathrm{kcal} / \mathrm{kg})$ and fat-free mass $(771 \mathrm{kcal} / \mathrm{kg})$. TDEE, total daily energy expenditure. $P$ represents the statistical significance of the group comparison, tested using linear mixed effect models with group used as a covariate. $P \leq 0.05$ is considered statistically significant. $P$ values in bold are significant. $\mathrm{A}, \mathrm{B}$ Shared letters indicate no significant differences between groups in post hoc comparison. 

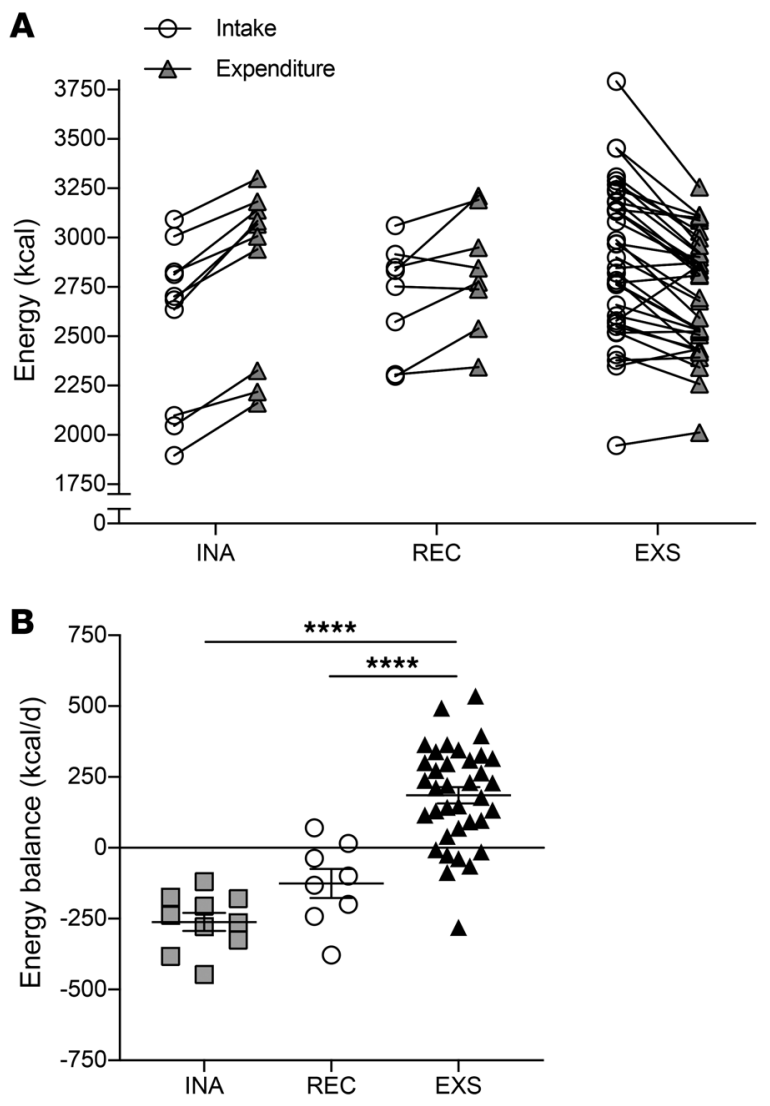

tinent to body composition and energy balance phenotyping. The proportion of women with recommended, excess, and inadequate weight gain in our study is consistent with epidemiological data in that two-thirds of women had excess gestational weight gain (2). Our study has 4 major findings that inform management of pregnant women with obesity. First, the recommended rate of weight gain was achieved by the gain of fat-free mass alone (e.g., fetus, uterus, blood volume, breast tissue). Second, dietary intake must not exceed energy expenditure during pregnancy, and weight gain as recommended by the Institute of Medicine is achieved if energy intake is maintained but not increased from early pregnancy throughout the second and third trimesters. The increased energy demand of pregnancy and by the infant is compensated for by the mobilization of maternal fat mass. Third, energy imbalance determines weight gain, without influence of diet, physical activity or metabolic biomarkers. Fourth, weight maintenance during obese pregnancy, as suggested by recent epidemiological studies, requires approximately $10 \%$ energy deficit.

First, the Institute of Medicine weight gain recommendations were achieved by the accumulation of fat-free mass alone. The gain in fat-free mass, which includes tissue growth (e.g., fetus, placenta, breast, and uterus) and fluid expansion (e.g., blood volume, intracellular, and extracellular fluid) (19), was similar among the weight gain groups. Energy balance was not associated with nonfat tissue accumulation, and therefore we hypothesize that interventions designed to modify energy balance would not affect weight gain in these compartments. To infer causality from this association requires evidence from a prospective intervention study. In contrast, fat mass gain was variable and thus is more like-
Figure 3. Energy intake, energy expenditure and energy balance. (A) Energy intake (energy intake-balance method) and energy expenditure (doubly labeled water method) are presented for each individual and as mean \pm SEM for 3 groups of women classified according to gestational weight gain as inadequate (in gray, INA, $<170 \mathrm{~g} /$ week, $n=10$ ), recommended (in white, REC, $\geq 170 \mathrm{~g} /$ week and $<270 \mathrm{~g} /$ week, $n=8$ ), and excessive (in black, EXS, $\geq 270 \mathrm{~g} /$ week, $n=36$ ) by the 2009 Institute of Medicine guidelines. (B) Individual and mean \pm SEM energy balance are presented as the difference in intake and expenditure for the 3 categories of gestational weight gain. Differences among weight gain groups were tested using linear mixed effect models with group used as a covariate. ${ }^{* * *} P<0.001$ of the post-hoc comparison between groups of the linear mixed model.

ly modifiable by interventions. Achieving the recommended rate of weight gain required that fat mass be reduced.

Second, the recommended rate of weight gain in obese pregnancies was accomplished when the daily energy intake throughout the second and third trimesters did not exceed energy expenditure. Our data suggest that pregnant women with obesity should not consume additional energy when pregnant. The energy requirement for fetal development was compensated by mobilization of maternal fat mass with no adverse effects in maternal or fetal outcomes observed. This observation challenges current advice by the Institute of Medicine and American College of Obstetrician and Gynecologists for women with obesity, which is to consume an additional $200-300 \mathrm{kcal} / \mathrm{d}$ during the second and third trimesters for optimal weight gain $(1,12)$. Consuming surplus energy throughout the second and third trimesters, even in these small amounts $(\sim 200 \mathrm{kcal} / \mathrm{d})$ produced excess gestational weight gain. Dietary needs should be estimated on a per patient basis using an energy requirement model specific for women with obesity early in pregnancy, or clinicians should simply advise patients to not increase dietary energy intake (20).

Third, no differences in physical activity, metabolic biomarkers, dietary intake, and eating behavior constructs were observed among the Institute of Medicine weight gain groups. Our study was not powered to detect differences in these factors and could be prone to type 1 error. Nevertheless, this suggests that the impact of a lifestyle modification intervention in pregnancy depends on its ability to modify energy balance. By our calculations, such trials aimed to achieve a reduction in energy intake of $7 \%-37 \%$ in the second and third trimesters (21-25). Importantly, the achieved differences in energy intake between the intervention and control groups, as estimated by the regression equation, were only small. Estimates of energy intake based on observed weight gain from published lifestyle intervention studies showed that interventions reduced energy intake by $-140 \mathrm{kcal} / \mathrm{d}(\sim 5 \%)$ in comparison with the control groups. Recommended weight gain was only achieved by 2 studies and, of note, our model estimates that women in these interventions increased energy intake during pregnancy by less than $100 \mathrm{kcal} / \mathrm{d}(23,24)$.

Past trials prescribing energy intake targets achieved the largest effect sizes (mean difference between intervention and control group) for modifying weight gain for women with obesity (21-25). Of the studies that examined modifying energy intake, most (2124) but not all (25) showed that dietary modification reduced one or more adverse pregnancy outcomes. However, the studies were powered to detect intervention effects on weight gain; hence, the 

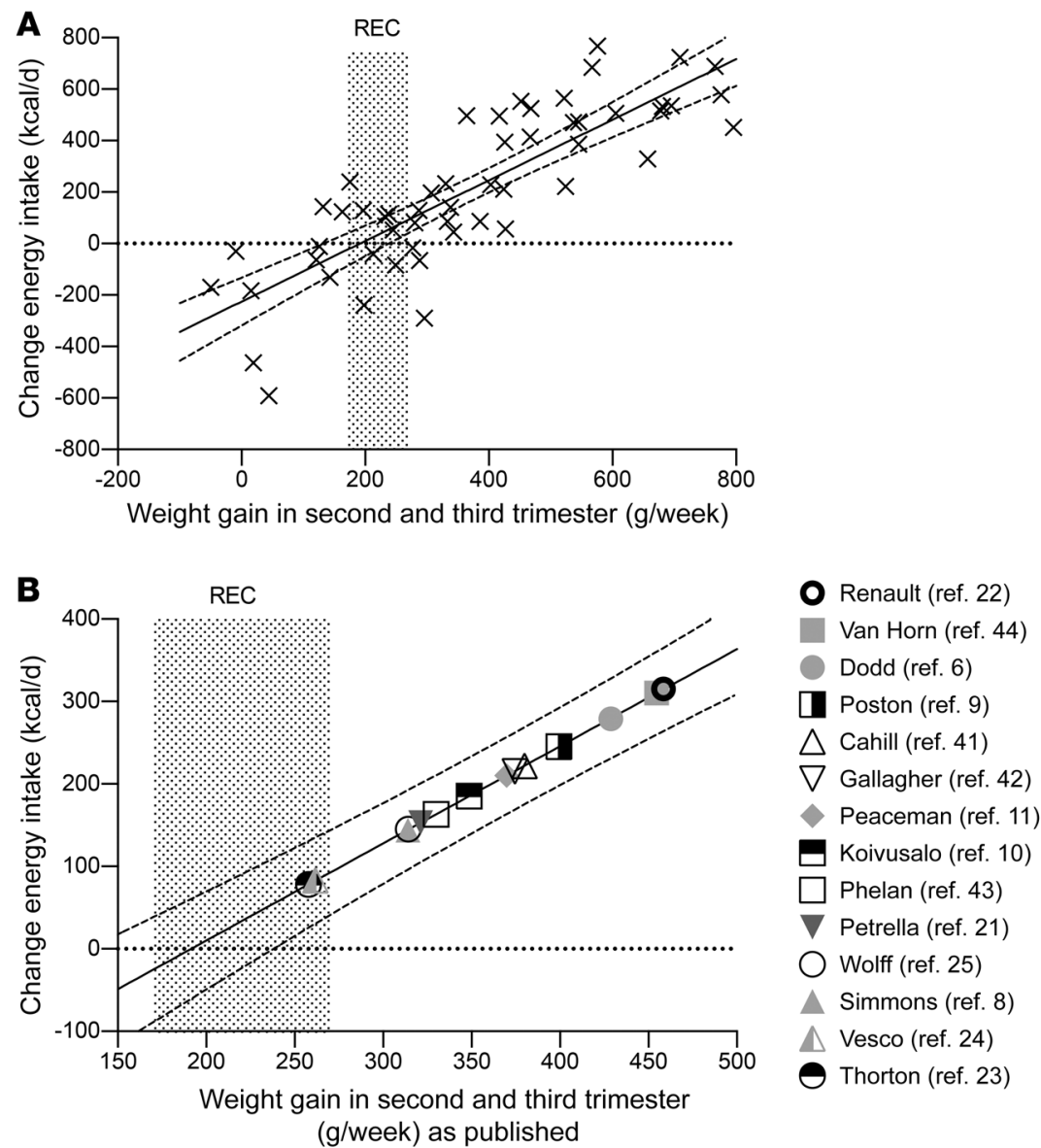

Figure 4. Change in energy intake estimates for gestational weight gain. Association between gestational weight gain and change in energy intake (Change El), calculated as calculated energy intake during pregnancy minus measured baseline total daily energy expenditure for each individual in the present study $(\mathbf{A}, n=54)$, and for intervention groups in lifestyle intervention studies for pregnant women with obesity (B, by first author). The association is defined by measured data in this study and defined as Change El [kcal $/ \mathrm{d}]=1.178$ $\pm 0.108 *$ GWG [g/week] $-225 \pm 46 ; R^{2}=0.70$, in which Change $\mathrm{El}$ is change in energy intake relative to early pregnancy total daily energy expenditure and CWG is gestational weight gain during the second and third trimesters. The regression line is presented with $95 \%$ confidence intervals. REC, recommended gestational weight gain (170-270 g/week). sample sizes were often too small to demonstrate intervention efficacy for improvements in other pregnancy outcomes. Conversely, those trials powered for pregnancy outcomes did not target energy intake or energy balance per se, and thereby achieved only modest reductions in energy intake and weight gain, and failed to modify adverse pregnancy and infant outcomes $(6,8-11)$. Appropriately powered studies designed to improve weight gain and pregnancy outcomes through evidence-based energy intake targets remain to be undertaken for women with obesity.

Finally, our study suggests that lifestyle interventions designed to achieve weight maintenance throughout pregnancy $(<2.5 \mathrm{~kg})$ require a $9 \%$ energy deficit. This finding is relevant to translate new recommendations from epidemiological studies that suggest that weight maintenance may be required to improve outcomes for pregnant women with obesity $(26,27)$. Advocating weight maintenance for pregnant women with obesity is however too premature until long-term effects on offspring development have been determined.

This study excels for its rigorous, objective methods. However, the approaches used in the study are limited to small, extensively phenotyped cohorts. Thus, our study is unique in its ability to explain how the current recommendations for weight gain can be achieved, and to demonstrate that excess gestational weight gain in women with obesity is due to differences in energy balance, which was not associated with differences or changes in metabolic determinants. The sample size is, however, too small to exclude the possibility of false-negative findings related to pregnancy, delivery, and fetal outcomes, or effect-modification by covariates, including demographic, behavioral, or metabolic factors. The next step is to evaluate the implementation of these new energy intake targets in appropriately powered studies and to understand the effects of maternal energy restriction on outcomes for women with obesity and their babies. Strategies to induce an energy gap may include attenuating the observed declines in physical activity and diet quality (28).

Using objective measures of energy balance, this study challenges current practice and argues that women with obesity should not be advised to consume additional energy during pregnancy as currently recommended. Preserving physical activity and improving diet quality may offer additional strategies to achieve current gestational weight gain recommendations, but only if an energy deficit of $\sim 100 \mathrm{kcal} / \mathrm{d}$ is achieved. More stringent recommendations for weight gain during obese pregnancy $(<2.5 \mathrm{~kg})$ would require deficits of approximately $10 \%$.

\section{Methods}

\section{Design and subjects}

MomEE (ClinicalTrials.gov, NCT01954342) was a prospective, observational cohort study conducted between February 2015 and January 2017 at Pennington Biomedical Research Center in Baton Rouge, Louisiana. Women were followed prospectively across pregnancy with outcome assessments measured in specialized clinic visits between 13-16 weeks and 35-37 weeks to encompass the second and third trimesters of gestation. Gestational weight gain per week was calculat- 
ed, and using the 2009 Institute of Medicine criteria (1), women were classified as having recommended (REC, 170-270 g/week), excess (EXS, $\geq 270 \mathrm{~g} /$ week), or inadequate (INA, $<170 \mathrm{~g} /$ week) weight gain. The primary outcome was daily energy intake throughout the second and third trimesters objectively assessed by the energy intake-balance method (29). Energy intake was calculated as the sum of mean energy expenditure across pregnancy (doubly labeled water) and energy deposition in fat and fat-free tissues (3-compartment model by plethysmography and isotope dilution) measured across the same observation period $(29,30)$. Secondary outcomes were changes in physical activity by accelerometry (31), energy expenditure during sleep by whole-body calorimetry (32), metabolic biomarkers, dietary intake by remote food photography $(33,34)$, and eating behavior constructs by validated questionnaires. Pregnancy, delivery, and infant outcomes were obtained from prenatal and delivery records.

Seventy-two pregnant women with obesity $\left(\mathrm{BMI} \geq 30 \mathrm{~kg} / \mathrm{m}^{2}\right.$ at $\leq 15$ weeks of gestation) were enrolled. Participants were required to be $18-40$ years old and pregnant with a singleton gestation confirmed by ultrasound ( $\geq 6$ weeks gestation). To study women with obesity who were otherwise healthy, participants were excluded for smoking, alcohol intake, drug use (prescription or recreational), hypertension ( $>160 / 110 \mathrm{mmHg}$ ), diabetes (HbA1c $\geq 6.5 \%$ ), severe anemia $(\mathrm{Hb}<8 \mathrm{~g}$ / $\mathrm{dL}$ and/or Hct $<24 \%$ ), and for factors with the possibility of abnormally affecting gestational weight gain or adherence to the study protocol (e.g., preeclampsia, bed rest prescription) (20). The primary care obstetric provider and medical investigator provided medical clearance for participation. Women did not receive specific advice on diet or activity but were informed about the Institute of Medicine guidelines on healthy gestational weight gain.

\section{Primary outcomes}

Full descriptions of the study procedures have been previously described (20,30, 34-36). In brief, at each assessment, participants presented to the research center for 2 outpatient visits and an overnight stay in a metabolic chamber (32). Body weight was measured fasted in a gown (with gown weight subtracted), and rate of gestational weight gain was calculated as change in measured body weight from early to late pregnancy, expressed in grams per week. Body fat mass was calculated using body weight, body volume by plethysmography (BODPOD, COSMED) and body water (mean estimate of using zero-intercepts of ${ }^{2} \mathrm{H}$ and ${ }^{18} \mathrm{O}$-isotopes) (37). Fat-free mass was calculated as body weight minus fat mass. Energy deposition was calculated assuming that $1 \mathrm{~kg}$ of fat is equivalent to $9500 \mathrm{kcal}$ and $1 \mathrm{~kg}$ of fat-free mass is equivalent to $771 \mathrm{kcal}(29,38)$. Free-living energy expenditure (TDEE) was measured over 7 days by doubly labeled water (1.25 g of $10 \%$ enriched $\mathrm{H}_{2}{ }^{18} \mathrm{O}$ and $0.10 \mathrm{~g}$ of $99.9 \%$ enriched ${ }^{2} \mathrm{H}_{2} \mathrm{O}$ per kg body weight) (20). TDEE across the observation period was calculated as the mean of the TDEE measured in early and late pregnancy. The primary outcome energy intake was calculated as sum of energy deposition and the mean energy expenditure across pregnancy.

\section{Secondary outcomes}

Energy metabolism was measured in the metabolic chamber. After participants ate a standard dinner at 1900 hours providing $30 \%$ of the estimated daily energy requirements (29) as $30 \%$ fat, $55 \%$ carbohydrate, and $15 \%$ protein, questionnaires were completed. Lights (including electronic screens) were off between 2230 hours and
0600 hours. Sleeping energy expenditure was the mean expenditure between 0200 hours and 0500 hours when activity (measured by infrared sensors) was $<1 \%$ per minute, extrapolated to 24 hours (20). Adaptive thermogenesis is the difference between measured sleeping energy expenditure and the adjusted value. Upon waking, participants emptied their bladders and laid awake and supine on the bed for a 30-minute measurement of resting metabolic rate. Physical activity was assessed as physical activity level calculated as TDEE divided by resting metabolic rate as well as mean amplitude deviation by accelerometry (ActiGraph GT3X+) (31). Participants wore the accelerometer on the nondominant wrist during the 7-day doubly labeled water assessment. Simultaneously, diet quality was assessed using a validated SmartIntake smartphone application $(33,34)$. Eating behavior constructs were assessed using validated questionnaires as described (39). Fetal weight was estimated by 3D ultrasound with measurements of head circumference, biparietal diameter, abdominal circumference, and femoral length and volume (40), obtained by the same sonographer.

\section{Estimate of change in energy intake for gestational weight gain}

To estimate the change in energy intake needed for a certain gestational weight gain, we used data from the cohort to generate the following linear regression equation to estimate the energy intake from energy deposition (i.e., gestational weight gain): Change $\mathrm{EI}[\mathrm{kcal} / \mathrm{d}]=$ $1.178 \pm 0.108^{*} \mathrm{GWG}$ [g/week] $-225 \pm 46 ; \mathrm{R}^{2}=0.70$. In our cohort, we estimated change in energy intake as energy intake during pregnancy minus early pregnancy energy expenditure, assuming energy balance in early pregnancy, and gestational weight gain per week, as previously described in "Design and subjects." Using the regression equation, gestational weight gain reported in previously published lifestyle intervention trials was used to compute the change in energy intake during pregnancy $(6,8-11,21-25,41-44)$.

\section{Statistics}

Power calculation. Using our previous assessment of energy intake in a predominantly nonobese cohort (38), we conducted an a priori sample size analysis which assumed the following: $\beta \geq 0.80, \alpha=0.05$, a $700 \mathrm{kcal} / \mathrm{d}$ SD for energy intake, and that the proportion of women with excess gestational weight gain would be $66 \%$ (1). Accordingly, a minimum of 51 subjects was required to detect a difference in energy intake of $\pm 520 \mathrm{kcal} / \mathrm{d}$. To achieve sufficient sample size considering miscarriages $(n=3)$, preterm deliveries ( $<37$ weeks, $n=3$ ), and attrition $(n=5), 72$ women were enrolled.

Differences between weight gain groups were tested using linear mixed effect models with group used as a covariate. With change from early pregnancy values as the outcome, differences between weight gain groups were tested using linear mixed effect models with group and the initial observation (i.e., between 13-16 weeks gestation) used as a covariate. Additional covariates tested included maternal age, race, parity, and fetal sex, but inclusion of these did not change the outcomes of the analysis. Final models are therefore presented without these additional covariates. Only if a main effect of the weight gain groups was significant, post hoc comparisons were assessed. A $P$ value less than 0.05 was considered significant. Reported $P$ values in the manuscript refer to the pairwise post hoc comparison with the group with recommended weight gain (REC), unless indicated otherwise. Analyses were carried out using SAS, Version 9.4 (SAS Institute). Finally, a 
sensitivity analysis was performed with the 2 women with the highest BMI $\left(46.0\right.$ and $\left.57.1 \mathrm{~kg} / \mathrm{m}^{2}\right)$ removed. While the difference in weight and BMI noted for the INA group in early pregnancy was no longer significant, the results of the primary outcomes remained unchanged.

\section{Study approval}

The protocol was approved by the Institutional Review Board at Pennington Biomedical Research Center and all participants provided written informed consent prior to initiation of study procedures. This study is registered clinical trial at ClinicalTrials.gov (NCT01954342).

\section{Author contributions}

JM, LAG, MSA, ER, and LMR conceived the experiments and designed the study. JM, MSA, ADA, LAG, PMV, and DSH carried out experiments. JM, RAB, DMT, ER, and LMR analyzed data. JM and LMR drafted the manuscript. All authors had final approval of the submitted and published versions. LMR had full access to all the data in the study and had final responsibility for the decision to submit for publication.

\section{Acknowledgments}

We would like to acknowledge administrative support from Elizabeth Sutton, Kelsey Olson, Alexandra Beyer, Alexis O'Connell, and Natalie Comardelle, and technical assistance of Jennifer Rood, Owen Carmichael, Kori Murray, Kevin McKlveen, Loren Cain, Kimberly Landry, Stephen Lee, Melissa Erickson, and Brian Gilmore (all Pennington Biomedical Research Center. This study was funded by the National Institutes of Health (R01DK099175 to LMR), with additional support by Clinical Research Cores at Pennington Biomedical Research Center (U54GM104940 and P3ODK072476). We greatly appreciate the recruitment and retention support from Ralph Dauterive, Evelyn Griffin (Ochsner Health System), and Evelyn Hayes (Baton Rouge General Medical Center). Above all, we thank the participants for allowing us to follow their pregnancies.

Address correspondence to: Leanne M. Redman, 6400 Perkins Road, Baton Rouge, Louisiana 70808, USA. Phone: 225.763.0947; 225.763.3022; Email: leanne.redman@pbrc.edu.
1. Institute of Medicine (US) National Research Council (US) Committee to Reexamine IOM Pregnancy Weight Guidelines. In: Rasmussen KM, Yaktine AL, eds. Weight Gain During Pregnancy: Reexamining the Guidelines. Washington (DC); 2009.

2. Deputy NP, Sharma AJ, Kim SY, Hinkle SN. Prevalence and characteristics associated with gestational weight gain adequacy. Obstet Gynecol. 2015;125(4):773-781.

3. Flegal KM, Kruszon-Moran D, Carroll MD, Fryar CD, Ogden CL. Trends in obesity among adults in the United States, 2005 to 2014. JAMA. 2016;315(21):2284-2291.

4. Catalano PM, et al. Perinatal risk factors for childhood obesity and metabolic dysregulation. Am J Clin Nutr. 2009;90(5):1303-1313.

5. Mamun AA, Kinarivala M, O'Callaghan MJ, Williams GM, Najman JM, Callaway LK. Associations of excess weight gain during pregnancy with long-term maternal overweight and obesity: evidence from 21 y postpartum follow-up. Am J Clin Nutr. 2010;91(5):1336-1341.

6. Dodd JM, et al. Antenatal lifestyle advice for women who are overweight or obese: LIMIT randomised trial. BMJ. 2014;348:g1285.

7. Simmons D, et al. Results from a European multicenter randomized trial of physical activity and/ or healthy eating to reduce the risk of gestational diabetes mellitus: The DALI Lifestyle Pilot. Diabetes Care. 2015;38(9):1650-1656.

8. Simmons D, et al. Effect of physical activity and/or healthy eating on GDM risk: The DALI Lifestyle Study. JClin Endocrinol Metab. 2017;102(3):903-913.

9. Poston L, et al. Effect of a behavioural intervention in obese pregnant women (the UPBEAT study): a multicentre, randomised controlled trial. Lancet Diabetes Endocrinol. 2015;3(10):767-777.

10. Koivusalo SB, et al. Gestational diabetes mellitus can be prevented by lifestyle intervention: the Finnish gestational diabetes prevention study
(RADIEL): a randomized controlled trial. Diabetes Care. 2016;39(1):24-30.

11. Peaceman AM, et al. Lifestyle interventions limit gestational weight gain in women with overweight or obesity: LIFE-Moms prospective meta-analysis. Obesity (Silver Spring). 2018;26(9):1396-1404.

12. American College of Obstetricians Gynecologists. Nutrition in Pregnancy. In: Your Pregnancy and Childbirth: Month to Month, Sixth Edition. Washington, DC: ACOG; 2016:313-327.

13. Institute of Medicine. Energy. In: Dietary Reference Intake for Energy, Carbohydrate, Fiber, Fat, Fatty Acids, Cholesterol, Protein, and Amino Acids. Washington, DC: The National Academies Press; 2005:107-264.

14. Butte NF, Wong WW, Treuth MS, Ellis KJ, O'Brian Smith E. Energy requirements during pregnancy based on total energy expenditure and energy deposition. Am J Clin Nutr. 2004;79(6):1078-1087.

15. Goldberg GR, et al. Longitudinal assessment of energy expenditure in pregnancy by the doubly labeled water method. Am JClin Nutr. 1993;57(4):494-505.

16. Kopp-Hoolihan LE, van Loan MD, Wong WW, King JC. Longitudinal assessment of energy balance in well-nourished, pregnant women. Am J Clin Nutr. 1999;69(4):697-704.

17. Evenson KR, Chasan-Taber L, Symons Downs D, Pearce EE. Review of self-reported physical activity assessments for pregnancy: summary of the evidence for validity and reliability. Paediatr Perinat Epidemiol. 2012;26(5):479-494.

18. Thomas DM, et al. Relationships between misreported energy intake and pregnancy in the pregnancy, infection and nutrition study: new insights from a dynamic energy balance model. Obes Sci Pract. 2016;2(2):174-179.

19. Lederman SA, Paxton A, Heymsfield SB, Wang J, Thornton J, Pierson RN. Body fat and water changes during pregnancy in women with different body weight and weight gain. Obstet Gynecol.
1997;90(4 Pt 1):483-488.

20. Most J, et al. Energy expenditure in pregnant women with obesity does not support energy intake recommendations. Obesity (Silver Spring). 2018;26(6):992-999.

21. Petrella E, et al. Gestational weight gain in overweight and obese women enrolled in a healthy lifestyle and eating habits program. J Matern Fetal Neonatal Med. 2014;27(13):1348-1352.

22. Renault KM, et al. The treatment of obese pregnant women (TOP) study: a randomized controlled trial of the effect of physical activity intervention assessed by pedometer with or without dietary intervention in obese pregnant women. Am JObstet Gynecol. 2014;210(2):134.e1-134.e9.

23. Thornton YS, Smarkola C, Kopacz SM, Ishoof SB Perinatal outcomes in nutritionally monitored obese pregnant women: a randomized clinical trial. J Natl Med Assoc. 2009;101(6):569-577.

24. Vesco KK, et al. Efficacy of a group-based dietary intervention for limiting gestational weight gain among obese women: a randomized trial. Obesity (Silver Spring). 2014;22(9):1989-1996.

25. Wolff S, Legarth J, Vangsgaard K, Toubro S, Astrup A. A randomized trial of the effects of dietary counseling on gestational weight gain and glucose metabolism in obese pregnant women. Int JObes (Lond). 2008;32(3):495-501.

26. Bodnar LM, et al. Low gestational weight gain and risk of adverse perinatal outcomes in obese and severely obese women. Epidemiology. 2016;27(6):894-902.

27. Durie DE, Thornburg LL, Glantz JC. Effect of second-trimester and third-trimester rate of gestational weight gain on maternal and neonatal outcomes. Obstet Gynecol. 2011;118(3):569-575.

28. Tsigga M, Filis V, Hatzopoulou K, Kotzamanidis C, Grammatikopoulou MG. Healthy eating index during pregnancy according to pre-gravid and gravid weight status. Public Health Nutr. 2011;14(2):290-296.

29. Thomas DM, et al. Dynamic energy-balance model predicting gestational weight gain. $A m J$ 


\section{CLINICAL MEDICINE}

Clin Nutr. 2012;95(1):115-122.

30. Most J, et al. Is energy balance in pregnancy involved in the etiology of gestational diabetes in women with obesity? Cell Metab. 2019;29(2):231-233.

31. Vähä-Ypyä H, Vasankari T, Husu P, Suni J, Sievänen H. A universal, accurate intensitybased classification of different physical activities using raw data of accelerometer. Clin Physiol Funct Imaging. 2015;35(1):64-70.

32. Lam YY, Ravussin E. Analysis of energy metabolism in humans: a review of methodologies. Mol Metab. 2016;5(11):1057-1071.

33. Martin CK, et al. Validity of the remote food photography method (RFPM) for estimating energy and nutrient intake in near real-time. Obesity (Silver Spring). 2012;20(4):891-899.

34. Most J, et al. Food photography is not an accurate measure of energy intake in obese, pregnant women. J Nutr. 2018;148(4):658-663.
35. Most J, et al. Propensity for adverse pregnancy outcomes in African-American women may be explained by low energy expenditure in early pregnancy. Am JClin Nutr. 2018;107(6):957-964.

36. Most J, Redman LM. Does energy expenditure influence body fat accumulation in pregnancy? Am JObstet Gynecol. 2019;220(1):119-120.

37. Most J, Marlatt KL, Altazan AD, Redman LM. Advances in assessing body composition during pregnancy. Eur JClin Nutr. 2018;72(5):645-656.

38. Gilmore LA, Butte NF, Ravussin E, Han H, Burton JH, Redman LM. Energy intake and energy expenditure for determining excess weight gain in pregnant women. Obstet Gynecol. 2016;127(5):884-892.

39. Most J, Rebello CJ, Altazan AD, Martin CK, Amant MS, Redman LM. Behavioral determinants of objectively assessed diet quality in obese pregnancy. Nutrients. 2019;11(7):E1446.

40. Lee W, et al. New fetal weight estimation models using fractional limb volume. Ultrasound Obstet Gynecol. 2009;34(5):556-565.

41. Cahill AG, et al. Weight control program and gestational weight gain in disadvantaged women with overweight or obesity: a randomized clinical trial. Obesity (Silver Spring). 2018;26(3):485-491.

42. Gallagher D, et al. Greater neonatal fat-free mass and similar fat mass following a randomized trial to control excess gestational weight gain. Obesity (Silver Spring). 2018;26(3):578-587.

43. Phelan S, Phipps MG, Abrams B, Darroch F, Schaffner A, Wing RR. Randomized trial of a behavioral intervention to prevent excessive gestational weight gain: the Fit for Delivery Study. Am J Clin Nutr. 2011;93(4):772-779.

44. Van Horn L, et al. Dietary approaches to stop hypertension diet and activity to limit gestational weight: maternal offspring metabolics family intervention trial, a technology enhanced randomized trial. Am J Prev Med. 2018;55(5):603-614. 